general population of up to $2 \%$. There has been growing evidence supporting its familial predisposition with an autosomal dominant pattern of inheritance. It is often associated with ascending aortic dilatation and dissection, occurring at a younger age than in patients with idiopathic aortic aneurysms. BAV disease carries a 6\% lifetime risk of aortic dissection, 9 times higher than that of the general population. Thus, the presence of BAV and dilatation of the ascending aorta requires regular monitoring with a view to timely pre-emptive surgery. Current ACC/AHA guidelines state that echocardiographic screening of first degree relatives of patients with BAV is recommended. This, however, to our knowledge, is not routinely done within the UK.

Methodology and Results We set out to explore the practicalities of running a routine echocardiographic screening programme for first degree relatives of patients with BAV disease. We identified a total of 47 patients who had undergone aortic valve surgery performed by the same Consultant Cardiothoracic Surgeon in the context of BAV disease in the period May 2007-September 2009. Screening of first degree relatives was offered to these patients. 24 patients (51\%) gave us information regarding family members who would like to attend for an echocardiogram. A total of 75 first degree relatives were referred-an approximate average of 3 per patient. Out of these, 52 relatives $(70 \%)$ actually attended for an appointment. The remainder did not undergo testing with us as they either lived in a different geographic region or expressed a personal preference not to be scanned at this time. The incidence of newly diagnosed bicuspid aortic valve disease in our cohort of first degree relatives was $8 \%$ (4 out of 52 relatives). One of these asymptomatic individuals had a significant ascending aortic aneurysm, which required prompt surgery. Among the relatives of the 24 index patients, there were a total of 8 cases (3: 1 ratio) of bicuspid aortic valve disease-either known or newly diagnosed via screening.

Conclusions There is a relatively high prevalence and incidence of bicuspid aortic valve disease among first degree relatives of patients with this common congenital cardiac abnormality. Routine echocardiographic screening should be offered to these families. Implementing such a programme is limited by adequate motivation to attend for a screening test if well, and by varying clinical practice in different geographic regions. Patients with bicuspid aortic valve disease should be made aware of its familial pattern of inheritance and screening of their first degree relatives should be actively pursued in order to reduce the potential morbidity and mortality associated with this condition and its related aortopathy.

\section{A CITED2->VEGFA PATHWAY COUPLES MYOCARDIAL AND CORONARY VASCULAR GROWTH IN THE DEVELOPING MOUSE HEART}

doi:10.1136/heartjnl-2011-300198.137

${ }^{1} S$ D Bamforth, 'S T MacDonald, 'J Braganca, ${ }^{1} \mathrm{C}-\mathrm{M}$ Chen, ${ }^{1} \mathrm{C}$ Broadbent, ${ }^{1} \mathrm{~J}$ E Schneider, ${ }^{2} \mathrm{R}$ Schwartz, ${ }^{1} \mathrm{~S}$ Bhattacharya. 'University of Oxford, Oxford, UK; ${ }^{2}$ Texas A\&M Health Science Centre, Houston, Texas, USA

Introduction Myocardial development is dependent on the concomitant growth of cardiomyocytes and a supporting vascular network. The coupling of myocardial and coronary vascular development is mediated in part by VEGFA signalling. Cited2 is a transcriptional cofactor that can inhibit hypoxia-activated transcription and also acts as a co-activator for transcription factors such as TFAP2. Genetic evidence indicates that Cited 2 is essential for cardiac left-right patterning via regulation of the Nodal-Pitx2c left-right patterning pathway. Zygotic and epiblastic deletion of Cited2 results in atrioventricular septation, outflow tract and aortic arch abnormalities, as well as left-right patterning defects such as right-isomerism. Cited2 is also essential for adrenal, neural crest, liver, lung, lens and placental development. However, the early requirement of Cited 2 in left-right patterning and placental development makes it difficult to identify a later specific role for Cited 2 in myocardial development. To overcome this problem we therefore investigated the role of Cited2 in the myocardium by conditional deletion in cardiomyocyte precursors. Methods Cited 2 was selectively deleted from cardiomyocytes by intercrossing mice transgenic for Cited 2 and Nkx2-5Cre. Embryos were collected and processed for analysis by histology, MRI, X-Gal staining, quantitative reverse transcriptase PCR (Q-RTPCR), chromatin immunoprecipitation and transient transfection assays.

Results The cardiomyocyte specific knockout of Cited 2 results in abnormal myocardial compact zone growth and ventricular septal defects. This is associated with a decreased ratio in the number of small vessels to large vessels, and a reduction in Vegfa expression. We also show that CITED2 is present at the Vegfa promoter in mouse embryonic hearts, and that it stimulates human VEGFA promoter activity in cooperation with TFAP2 transcription factors in transient transfection assays. However, we observed no change in the myocardial expression of the left-right patterning gene Pitx $2 c$, a known target of Cited2.

Conclusions The myocardial and capillary defects observed in myocardial loss of Cited 2 are not associated with Pitx $2 c$ deficiency and suggests that Cited 2 can cause myocardial and vascular defects via a mechanism that is distinct from its effect on the left-right patterning pathway. Our results delineate a novel mechanism of Vegfa regulation by CITED2 and TFAP2 transcription factors, and indicate that coupling of myocardial and coronary vascular growth in the developing mouse heart occurs, at least in part, through a Cited2->Vegfa pathway. This pathway may be targeted for the treatment of heart failure resulting from ischaemic heart disease.

\section{CELL-SPECIFIC ROLE OF NOX2 NADPH OXIDASE IN DEVELOPMENT OF ANGIOTENSIN II-INDUCED CARDIAC FIBROSIS IN VIVO}

doi:10.1136/heartjnl-2011-300198.138

${ }^{1} \mathrm{~S}$ Chaubey, ${ }^{1} \mathrm{C}$ E Murdoch, ${ }^{1} \mathrm{~A}$ Ivetic, ${ }^{1} \mathrm{~B}$ Yu, ${ }^{2} \mathrm{D}$ Vanhoutte, ${ }^{2} \mathrm{~S}$ Heymans, ${ }^{1} \mathrm{~A}$ Brewer, ${ }^{1} \mathrm{~A}$ M Shah. ${ }^{1}$ Kings College London BHF Centre of Excellence, London, UK; ${ }^{2}$ University Hospital Maastricht, Maastricht, The Netherlands

Introduction Mice globally deficient in Nox2 are protected against cardiac fibrosis in response to chronic AngII infusion even though the degree of hypertrophy was unaltered. The selective effect of Nox2 on fibrosis may reflect its activation in a non-cardiomyocyte cell type. We hypothesised that Nox2, which is expressed in endothelial cells and inflammatory cells, may be important for cardiac fibrosis in these cell types.

Methods To investigate the role of Nox2 in inflammatory cells, we generated chimeric mice by irradiation (10Gy, $15 \mathrm{~min}$ ) to deplete resident bone marrow cells, followed by bone marrow (BM) transplantation, using the following permutations: wild-type (WT) recipient with either $\mathrm{KO}$ or WT $\mathrm{BM}$, and $\mathrm{KO}$ recipients with WT BM. To assess the role of endothelial Nox2, we used transgenic mice with endothelial-specific overexpression of Nox2 (TG) utilising the tie2 promoter construct.

Result AngII (1.1 mg/kg/day, 14-day) infusion caused similar increase in systolic hypertension and cardiac hypertrophy in all 3 chimeric groups. However, cardiac fibrosis assessed by Sirius red staining was significantly lower in KO mice receiving WT BM $(0.5 \pm 0.1 \%)$ compared to the WT:WT group $(2.7 \pm 0.7 \%)$ or in WT receiving $\mathrm{KO} \mathrm{BM}(2.3 \pm 0.6 \%)$. These data suggested that resident Nox2-expressing cells are responsible for the protective effect observed in global Nox2 KO mice. TG mice developed the same level of systolic hypertension and hypertrophy as WT littermates after AngII infusion. However, the extent of cardiac fibrosis was significantly greater in TG than WT by $\sim 2$-fold $(\mathrm{p}<0.05)$. This was associated with a greater degree of infiltration by $\mathrm{CD} 45+$ 